\title{
Consciousness is Result of Ether Energy Resonant Stabilisation Process - EERSP
}

By Pavle Vesic BHEC, Belgrade, Serbia Abstract- In last fifty years, consciousness appeared to be the most intriguing scientific question. For most people, that question, does not exists. The fact that we are aware that we exist is something what is not questionable.

With the development of quantum mechanics, where the matter was described by wave function, life and accompanied processes were supposed to be mathematically describable. Most of living internal function were successfully described by temporary chemistry and physic but, consciousness phenomena was not explainable within that domain. David Chalmers posed the most difficulties consciousness question: hard problem.

This article, in short, describes consciousness as a result in ether energy resonant stabilization process (EERSP), based on ATOS (1) and Process physics (2).

GJHSS-A Classification: FOR Code: 190499

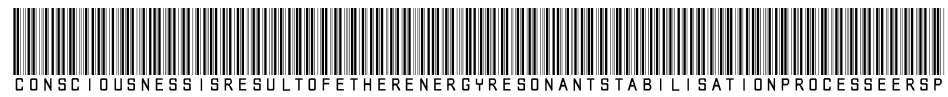

Strictly as per the compliance and regulations of:

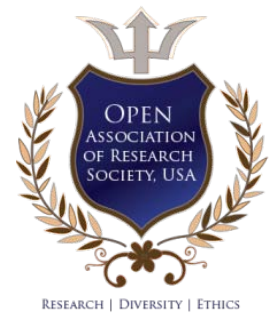

(C) 2020. Pavle Vesic BHEC, Belgrade, Serbia. This is a research/review paper, distributed under the terms of the Creative Commons Attribution-Noncommercial 3.0 Unported License http://creativecommons.org/licenses/by-nc/3.0/), permitting all noncommercial use, distribution, and reproduction in any medium, provided the original work is properly cited. 


\title{
Consciousness is Result of Ether Energy Resonant Stabilisation Process - EERSP
}

\author{
Pavle Vesic BHEC, Belgrade, Serbia
}

\begin{abstract}
In last fifty years, consciousness appeared to be the most intriguing scientific question. For most people, that question, does not exists. The fact that we are aware that we exist is something what is not questionable.

With the development of quantum mechanics, where the matter was described by wave function, life and accompanied processes were supposed to be mathematically describable. Most of living internal function were successfully described by temporary chemistry and physic but, consciousness phenomena was not explainable within that domain. David Chalmers posed the most difficulties consciousness question: hard problem.
\end{abstract}

This article, in short, describes consciousness as a result in ether energy resonant stabilization process (EERSP), based on ATOS (1) and Process physics (2).

\section{The Beginning}

B ased on documents (1) and (2), cosmos is closed system of infinite vibrating energy. One of cosmos formation theory is documented in (2), which bring us to the moment of space and time existence primary superimposition (1).

It is very important to stress the fact that in that moment THERE WAS NOT ANY POSSIBLE TEMPLATE which will point out even lightest direction sign (arrow) (3) of the matter formation.

Ether energy in primary superimposition state, started never ending creating process. That was the moment which, after long time, caused idea of God existence.

Elementary particles (electron and proton) were formed according to EERSP.

What is result of EERSP is documented in (4) where electron structure is brilliantly described:

This is a much more satisfactory and rigorous description of the electron and explanation of the Bohr radius. Rather than not knowing if the electron is a particle, wave, cloud, cavity, or "a point particle in a cloud of probable locations", it is best understood as a "precise toroidal volumetric flux structure" of energy resulting from two ambipolar longitudinal waves being superimposed upon and trapped with each other "that occupies the location of the entire "cloud".

EERSP acts NOW and will act forever. Two energy waves, which are named magnetic and electric, are pure ether energy in resonant state. Resonance is cosmic principle which generate stability: all particles in

Author: e-mail: pavlevesic@gmail.com cosmos have the same characteristics, all physical laws are the same, as well.

After elementary particles creation, matter was created under already described principle -EERSP.

Hydrogen atom consists of two elementary particles (electron and proton). Electron is relatively easy to describe, thanks to the position which he generally takes in matter (periodic table of elements). Proton constitutes atom core and, is not alone. He is accompanied with neutron (not elementary particle). WHY? According to EERSP, hydrogen atom cannot exists without neutron. Neutron role is to resolve all energy dynamic instability between electron and proton generated due to different energy space distribution. That is why during neutron time decomposition, energy equivalent to electron and proton are detected. Neutron is EERSP product, with dedicated role, which, after hydrogen atom decomposition, goes back to energy sea (5).

\section{EeRsp is Creativity Principle so LONGLY MANKIND SOUGHT}

Existing and new created matter can combine on practically infinite ways as far they obey EERSP. In one point of ever existing universe, the simplest life products appeared. Some of them, among other characteristics, were able to move, change position in space. In all phase of life evolution (and now), EERSP acts, on the very same way as in hydrogen atom neutron has been created. In every phase of life upraising, EERSP generated necessary products (life neutrons). It is extraordinary coincidence, that high level stability mechanism is materialized in brain cell named neuron. Neuron is the cell which is able to generate energy signals on ether level. According to the EERSP, it is the only way how higher level of matter can be brought to stable state. On the top of that, stabilization is needed constantly, what neuron cell in fact does. First neuron cell creation is equivalent to the first cosmic hydrogen neutron creation.

What happened after the first neuron cell appeared, thanks to the DNA "book kipping" ability, on the best way was described by Tomas Nagel:

"Each of our lives is a part of the lengthy process of the universe gradually waking up and becoming aware of itself". 
The first living creatures were stabilized by the first neuron cells, after cerebellum started to form. At the undefined point of the "lengthy process", our ancestors have had well developed cerebellum.

Third level of EERSP manifestation was brainstem formation, what was prerequisite for brain itself. In order to keep body under the full control, new brain function were generated and physically realized in now known brain structures (all the time we have to have DNA on mind).

Part of the brain where all communication between brain and body is performed is brainstem.

All two way signals of brain - body communication pass brainstem. Control energy information flux related to our existence is consciousness.

What is the highest level EERSP manifestation?

According to ATOS pure energy is primary source of everything. Energy entropy manifests itself constantly.

"On the adequate concepts of Life, the living and the universe An adequate concept of Life or of the living requires a fundamental understanding of what Life is, and this is only possible - we contend - when taken from the viewpoint of energy. The production of a body and a mind is first and foremost the cellular event that scopes and assembles the molecular machinery. This can only be grasped in terms of conjunctions of energy fluxes which permit the emergence of systems that are capable of autonomously increasing their internal energy to heighten their order - and by heightening their order. Stating this calls for explaining just what the parts are from the viewpoint of the whole, and the whole from the viewpoint of the parts. This dual movement is a reciprocal operation that yields results exactly contrary to what biocentrism suggests. Indeed, the viewpoint of Life can never be the viewpoint of the universe. On the basis of the Conservation Law of Physics, which is also the First Law of thermodynamics, the universe as a system cannot increase its energy content (there is no outside from which it can draw), whereas every living system functions on the basis of a steady increase of its energy content, and one that is effectuated at the expense of its surroundings. Life's particularity lies in the fact that it can increase its energy content over time, something that the universe cannot".

It is not easy to explain brain activity and diversity development vs energy consumption. According to EERSP, energy entropy on cosmic level, should be stable, what is source of long seeking teleology mentioned in Tomas Nagel Mind and Cosmos.

\section{Conclusion}

Energy flow manifested in brainstem, that is, information about everything what happens in our body and out of us, detected by our organs, we experience as CONSCIOUSNESS.

Consciousness is constant awareness of NOW existing information.

There is not any intermediary level between ether and us. What happens in realm of energy (ether) we directly experience as energy flow throw brainstem, i.e. we become conscious about that. That is explanation why all of us see the same colors, dimensions, and so on. There is no intermediary process of life comprehension. All living creatures are ON LINE, direct input- output devices, in ether realm.

Ether is absolute reality.

That is why many scientist and religious guru think that ether is equal to consciousness.

1. https://www.aetherometry.com/Aetherometry_Intro/V olume2B_Intro.pdf

2. https://arxiv.org/pdf/gr-qc/0203015.pdf

3. https://en.wikipedia.org/wiki/Mind_and_Cosmos

4. https://globaljournals.org/GJSFR_Volume18/6Revisiting-the-Bohr-Radius.pdf

5. https://www.youtube.com/watch? $v=L P h g D f T 4 Z p c$

Belgrade, 05.10.20. 